Editorial

\title{
Special Issue on Precision Risk Stratification-Based Screening in Colorectal Cancer
}

\author{
Alexander Stojadinovic ${ }^{1 凶}$, Itzhak Avital ${ }^{2 凶}$, Scott Steele $^{3^{凶}}$ \\ 1. Walter Reed National Military Medical Center, 8901 Wisconsin Avenue, Bldg. 9, Room 1272, Bethesda, MD 20889, USA; \\ 2. Bon Secours Cancer Institute, Richmond VA, USA; \\ 3. Department of Surgery, Uniformed Services University of the Health Sciences, Bethesda, MD, USA
}

$\triangle$ Corresponding author: Stojadinovicmd2011@gmail.com; Itzhak.Avital@gmail.com; harkersteele@me.com.

( ) Ivyspring International Publisher. This is an open-access article distributed under the terms of the Creative Commons License (http://creativecommons.org/ licenses/by-nc-nd/3.0/). Reproduction is permitted for personal, noncommercial use, provided that the article is in whole, unmodified, and properly cited.

Received: 2013.01.15; Accepted: 2013.02.05; Published: 2013.03.01

Cancer remains a formidable public health burden, as over half a million Americans succumb to this disease each year. The fight to vanquish cancer is stronger than ever. The pace of acquisition of new, cutting-edge scientific knowledge about the complex biology of cancer, and the development of technologies, targeted therapeutics and innovative approaches to prevent, detect and treat the disease have accelerated considerably over the past decade. Hence, in just over a century we have witnessed an extraordinary evolution of medical practice from seeking to understand disease in a physician-directed healthcare paradigm, to evidence-based medicine, and on to the modern era of emerging personalized, predictive, precision medicine (which applies predictive and prognostic biomarkers and patient-specific attributes using a "personalized lifespan approach").

During the past year we have witnessed not only a multi-faceted national effort to improve quality and value of cancer care aligned with the Institute for Healthcare Improvement's "triple aim" Initiative [improving the individual patient care experience, the health of populations, and thereby the efficiency and cost of care], but also realized several major clinical advances in cancer treatment [http://www.ihi. org/offerings/Initiatives/TripleAim/Pages/default. aspx]. In 2012 alone, the FDA approved seven new targeted systemic agents and expanded the anti-cancer indications for five existing agents. Noteworthy progress has been made in overcoming treatment resistance (combining targeted drugs, use of multi-targeted agents, targeting new pathways), understanding molecular drivers of cancer and tumor heterogeneity across the genomic landscape to include gene-environment interactions, advancing precision medicine, improving and enabling risk stratification-based screening and early detection.

To this latter point, a minimally invasive technique (flexible sigmoidoscopy) has been shown in a large randomized clinical trial to significantly reduce both incidence (by $21 \%$; relative risk, $0.79 ; 95 \%$ confidence interval $[\mathrm{CI}], 0.72$ to $0.85 ; \mathrm{P}<0.001)$ and overall colorectal cancer mortality (by $26 \%$; relative risk, 0.74 ; 95\% CI, 0.63 to 0.87; P<0.001) [N Engl J Med. 2012 Jun 21;366(25):2345-57]. This represents a major advance in cancer screening and early disease detection. It is decidedly poised to transform population-based screening, and to support concerted efforts to unravel socioeconomic disparities in cancer screening. What's more, this safe and effective screening approach will be used to educate screening populations about the importance of early detection, and could inspire wide-ranging compliance with recommended colorectal screening guidelines.

The current special edition of the Journal of Cancer is centered on colorectal cancer and it highlights advances made in the prevention, screening, early detection, and treatment of this disease. It communicates an ambitious vision aimed at conquering colorectal cancer, and provides recommendations for leveraging innovative technological advances, particularly those that provide insights into 
patient-specific tumor biology, genetic make-up and epigenetic regulation. It emphasizes the way forward through progress made in screening for colorectal cancer in an ethnically and socio-economically diverse population with equal access to care.

The special edition further emphasizes safe, effective, quality-based, innovative colorectal cancer care early detection and individualized precision medicine through Precision Risk Stratification-Based Screening. The principal aim of this risk identification and screening approach is to identify patients at risk for or diagnoses patients early in the natural history of colorectal cancer. It further defines evidence-based methods by which to avoid the toxicity of systemic therapy in appropriately risk-stratified patients identified as low risk for disease recurrence that are likely cured by surgery alone; appropriately classify patients with clinically latent or stable residual disease for whom systemic therapy can be withheld, and accurately recognize those high-risk node-negative patients with colorectal cancer or those with treatment-responsive disease likely to progress and treat them at the optimal time in the course of their disease. This special edition charts an ambitious and attainable way forward, and allows us ample basis by which to imagine a world without cancer. 